\title{
Psicooncología
}

ISSN: 1696-7240

\section{Experiencia del paciente: una nueva forma de entender la atención al paciente oncológico}

\author{
Carmen Yélamos ${ }^{1}$; Ana Sanz ${ }^{2}$; Ruth Marín ${ }^{3}$; Cristina Martínez-Ríos ${ }^{4}$
}

Recibido: 5 de enero de 2017 / Aceptado: 24 de febrero de 2018

Resumen: En el presente trabajo se analiza el concepto de Experiencia del Paciente (PX) así como las variables que contribuyen a la mejora del mismo. El objetivo es explicar su utilidad clínica en el ámbito de la salud, en concreto sobre la población oncológica, además de demostrar el papel activo que la Psicooncología puede tener en la implementación de esta estrategia, en relación directa con la humanización de la asistencia sanitaria. Para ello, se ha revisado la literatura con evidencia empírica existente hasta el momento y que sitúa la experiencia del paciente como un pilar indispensable a la hora de mejorar la calidad de la atención clínica, junto con la efectividad del tratamiento y la seguridad del paciente. Estos elementos presentan una interacción positiva entre ellos y están moderados por la comunicación médico-paciente, lo que evidencia la necesidad de desarrollar habilidades de comunicación por los profesionales en la práctica clínica, la coordinación de la atención sanitaria, el acceso a los servicios y la percepción del paciente respecto a la atención recibida. La experiencia del paciente resulta clínicamente relevante e influyente en la recuperación del estado de salud del paciente, así como constituye un cambio en la cultura sanitaria, cuyo protagonista es el paciente y en torno a él debe girar dicho cambio de manera multidisciplinar e integral por parte de los profesionales sanitarios implicados en su cuidado. Se concluye con una nueva línea de actuación del psicooncólogo en la experiencia del paciente para promover un servicio oncológico de calidad.

Palabras clave: Experiencia del paciente; comunicación; humanización.

\section{[en] Patient experience: A new way to understand care for the oncological patient}

\begin{abstract}
In the present paper, the concept of Patient Experience (PX) is analyzed, as well as the variables that contribute to its improvement. The aim is to explain its clinical utility in the field of health, specifically on the oncology population, in addition to demonstrating the active role that Psychooncology can have in the implementation of this strategy, in direct relation with the humanization of health care. For this, the literature has been reviewed with empirical evidence that has existed up to now and which places the patient's experience as an essential pillar when improving the quality of clinical care, together with the effectiveness of treatment and patient safety. These elements present a positive interaction between them and

1 Carmen Yélamos. GenesisCare. Madrid

E-mail: carmen.yelamos@genesiscare.es

2 Ana Sanz. GenesisCare. Madrid

E-mail: ana.sanz@genesiscare.es

3 Ruth Marín. GenesisCare. Castilla La Mancha

E-mail: ruth.marin@genesiscare.es

4 Cristina Martínez-Ríos. GenesisCare. Murcia

E-mail: cristina.martinez@genesiscare.es

* Dirección de correspondencia: Carmen Yélamos. GenesisCare C/ Emilio Vargas, 16 - 28043 Madrid. E-mail: carmen.yelamos@genesiscare.es
\end{abstract}


are moderated by the doctor-patient communication, which evidences the need to develop communication skills by professionals in clinical practice, the coordination of healthcare, access to services and perception of the patient regarding the care received. The patient's experience is clinically relevant and influential in the recovery of the health status of the patient, as well as constitutes a change in the health culture, whose protagonist is the patient and around him must turn this change in a multidisciplinary and integral way of the health professionals involved in their care. It is concluded with a new line of action of the psychooncologist in the patient's experience to promote a quality oncological service.

Keywords: Patient experience, communication, humanization.

Sumario. 1. 1. Introducción 2. Definición de experiencia del paciente 3. Cultura asistencial centrada en la experiencia del paciente a) Comunicación médico-paciente b) Coordinación de la atención prestada c) Accesibilidad a los servicios/atención d) Percepción del paciente respecto a la calidad de la atención e) Otros aspectos de la experiencia del paciente 4. La experiencia del paciente en el ámbito de la salud. utilidad clínica 5. Experiencia del paciente en población oncológica 6. ¿Es el enfoque centrado en la experiencia del paciente un nuevo desarrollo para la psicooncología? 7. Conclusiones 8 . Referencias bibliográficas.

Cómo citar: Yélamos C, Sanz A, Marín R, Martínez-Ríos C. Experiencia del paciente: una nueva forma de entender la atención al paciente oncológico. Psicooncología 2018;15:153-164. Doi: 10.5209/ PSIC.59184.

\section{Introducción}

En los últimos años, las percepciones de desempeño y calidad de las organizaciones de salud han comenzado a ir más allá de examinar la prestación de una excelente atención clínica. En este sentido, la experiencia del paciente en la institución durante la aplicación de los tratamientos está comenzando a considerarse como uno de los indicadores más relevantes de la atención sanitaria y, por tanto, de las políticas y decisiones que se deriven de la misma.

Actualmente existe confusión respecto al término patient experience (experiencia del paciente), probablemente debido a la novedad del mismo y la falta de instauración en el ámbito sanitario a nivel nacional. Por este motivo, se considera necesario definir este concepto, antes de señalar los beneficios de su inclusión en variables relativas a la salud y a la práctica clínica en el área sociosanitaria $\mathrm{y}$, de forma específica en la población oncológica. Finalmente se realizará una aproximación al papel que el psicooncólogo puede desempeñar dentro de esta nueva estrategia y ámbito de aplicación muy ligada a la humanización de la asistencia sanitaria.

\section{Definición de experiencia del paciente}

Hasta el 2014 no se encuentra en la literatura científica el primer artículo de revisión sobre el término Patient Experience $(P X)$, cuyos objetivos fueron identificar los elementos claves en su definición formal, así como promover y apoyar la misma en la investigación aplicada y en la práctica clínica. En este sentido, Wolf et al.(1) consideran que una de las definiciones más utilizada y aceptada a nivel mundial, es la desarrollada en el Beryl Institute ${ }^{(2)}$, que señala la experiencia del paciente como "la suma de todas las interacciones, configuradas por la cultura de una organización, que 
influyen en las percepciones del paciente, a lo largo de la continuidad del cuidado". El grupo de trabajo que diseñó esta definición indicó la necesidad de incluir cuatro elementos clave: las interacciones personales, la cultura de la organización, la percepción subjetiva del paciente y/o de la familia, así como el continuo cuidado.

Sin embargo, Wolf et al. ${ }^{(1)}$, a partir de una búsqueda exhaustiva en bases de datos y revistas científicas, identificaron una serie de puntos clave adicionales recogidos en la Tabla 1. Éstos ayudan a profundizar en la comprensión de la experiencia del paciente, de acuerdo con la naturaleza dinámica del concepto, además de recomendar una definición aplicable a los diferentes ámbitos de la atención clínica del paciente.

Tabla 1. Elementos claves para la definición de experiencia del paciente

\begin{tabular}{|c|c|}
\hline Elementos clave & Descripción. \\
\hline $\begin{array}{c}\text { Ideal de la atención } \\
\text { médica }\end{array}$ & $\begin{array}{l}\text { Cuatro necesidades emocionales básicas (confianza, integridad, } \\
\text { orgullo y pasión) aseguran que el paciente esté implicado y } \\
\text { comprometido con su atención médica }\end{array}$ \\
\hline $\begin{array}{c}\text { Continuidad en el } \\
\text { cuidado }\end{array}$ & $\begin{array}{l}\text { Calidad y valor de todas las interacciones (directas e indirectas, } \\
\text { clínicas y no clínicas) que abarcan todo el tiempo de la relación } \\
\text { médico-institución sanitaria representando un continuo. }\end{array}$ \\
\hline $\begin{array}{l}\text { Más allá de los } \\
\text { resultados de las } \\
\text { encuestas de los } \\
\text { sistemas de salud }\end{array}$ & $\begin{array}{l}\text { Las encuestas no integran la amplitud y la profundidad de la } \\
\text { experiencia del paciente. Debe incluirse el rendimiento racional } \\
\text { de la institución, las expectativas y aspectos emocionales del } \\
\text { paciente }\end{array}$ \\
\hline $\begin{array}{c}\text { Centrada en las } \\
\text { expectativas }\end{array}$ & $\begin{array}{l}\text { La experiencia del paciente comienza incluso antes de la } \\
\text { visita al centro, incluyendo las expectativas previas no sólo de } \\
\text { aspectos médicos; sino también técnicos, de accesibilidad o } \\
\text { limpieza. Hace referencia a la calidad de los servicios, la toma } \\
\text { de decisiones y la relación médico-paciente }\end{array}$ \\
\hline $\begin{array}{c}\text { Atención centrada en } \\
\text { el paciente }\end{array}$ & $\begin{array}{l}\text { Nueva cultura centrada en el paciente: atención competente y } \\
\text { de alta calidad, que demanda coordinación de los cuidados al } \\
\text { paciente y en la que todos los profesionales desempeñan un } \\
\text { papel relevante }\end{array}$ \\
\hline $\begin{array}{l}\text { Enfoque de cuidado } \\
\text { individualizado }\end{array}$ & $\begin{array}{l}\text { Marco de trabajo basado en el modelo biopsicosocial que implica } \\
\text { una serie de recomendaciones de las guías NICE: contemplar } \\
\text { al paciente como persona, incluir los requisitos esenciales en su } \\
\text { atención, adaptar los servicios sanitarios al paciente, favorecer } \\
\text { la continuidad asistencial y en las interacciones y fomentar el } \\
\text { papel activo del paciente durante su tratamiento }\end{array}$ \\
\hline Más que satisfacción & $\begin{array}{l}\text { La satisfacción con la atención recibida es un aspecto más de } \\
\text { la experiencia del paciente. La organización sanitaria debe } \\
\text { dar respuesta al paciente de acuerdo a las siguientes variables: } \\
\text { autonomía, dignidad, comunicación, confidencialidad, capacidad } \\
\text { de elegir, atención inmediata y calidad de los servicios }\end{array}$ \\
\hline
\end{tabular}


De acuerdo con la información revisada, los autores ${ }^{(1)}$ concluyen que la definición propuesta por el Beryl Institute, abarca de forma general el concepto de experiencia del paciente, ya que incluye elementos básicos como son: la suma de todas las interacciones del paciente con la institución, la influencia de la cultura de la organización en la experiencia vivida por el paciente, la importancia de considerar sus percepciones o valoraciones, así como identificar estas vivencias dentro de un continuo de la atención recibida. Aunque indican que existen tres aspectos, que no están contemplados en esta definición de forma explícita, y que sería necesario incluir para mejorar la comprensión del concepto y alcanzar una mayor aplicabilidad (Figura 1). Estos serían: la alianza y compromiso activo del paciente y la familia, la necesidad integral de centrarse en la persona y un reconocimiento del carácter amplio e integrado de la experiencia en general, incluyendo de esta forma aspectos relativos la calidad asistencial, seguridad terapéutica, etc.

Figura 1. Componentes de la definición de experiencia del paciente propuesta por Wolf et al. ${ }^{(1)}$

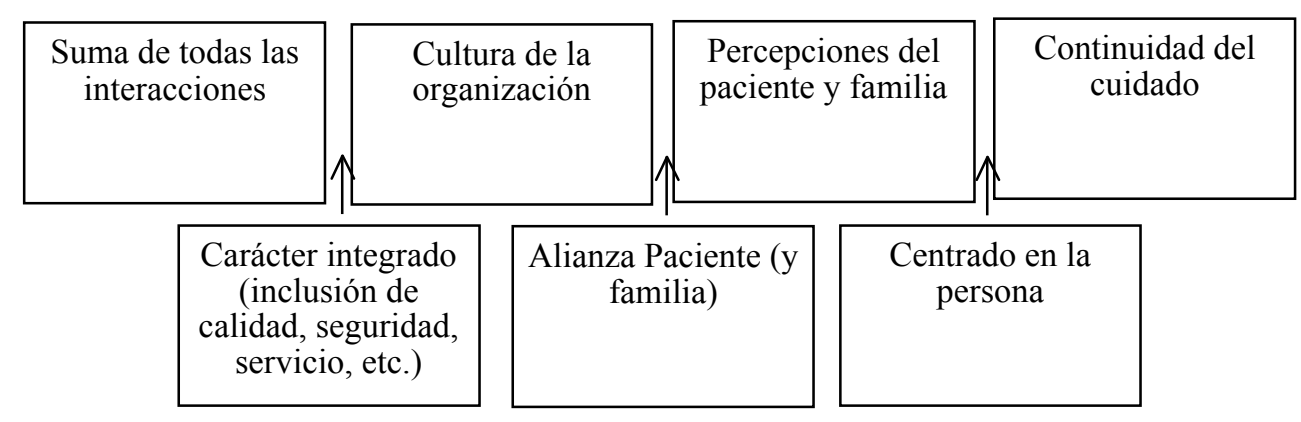

\section{Cultura asistencial centrada en la experiencia del paciente}

En un sistema sanitario, cuya legitimación social descansa en la fiabilidad, en la satisfacción y en la confianza, la experiencia general del paciente y de la población en su contacto con los servicios, es cada vez más importante, Mollica et al. ${ }^{(3)}$ revisan la literatura de experiencia del paciente y categorizan los artículos publicados en cinco áreas de interés que reflejan aspectos o factores críticos en la mejora de la experiencia del paciente: a) comunicación médico-paciente; b) coordinación de la atención prestada; c) accesibilidad a los servicios/atención; d) percepciones del paciente respecto a la calidad de la atención, y e) otros aspectos.

\section{a) Comunicación médico-paciente}

Los factores que contribuyen en mayor medida a la comunicación eficaz médicopaciente incluyen la información clara y oportuna, el apoyo emocional y la oportunidad para la toma de decisiones compartida. Mazor et al. ${ }^{(4)}$ señalan que los pacientes y los familiares son más conscientes y sensibles a dos funciones de la comunicación: intercambio de información (incluyendo los dominios de contenido, 
tiempo, suficiencia, claridad y exactitud) y fomento de la relación médico-paciente/ familia, incluyendo las dimensiones relacionadas con el trato interpersonal, compromiso e interés por conocer al paciente ${ }^{(5)}$. En general, los estudios indican que tanto el intercambio integral de información como una relación de calidad son las variables más importantes que influyen en la experiencia de los pacientes y sobre los resultados de salud ${ }^{(4)}$.

\section{b) Coordinación de la atención prestada}

La coordinación entre atención primaria y especializada, así como entre los distintos servicios dentro del área especializada son aspectos que influyen indudablemente en la experiencia del paciente ${ }^{(6-8)}$. Elementos que facilitan esta coordinación son: la facilidad en el itinerario de navegación del paciente, la comunicación efectiva y eficaz entre los profesionales implicados en el tratamiento del paciente, así como la habilidad de proporcionar información suficiente y oportuna a los pacientes ${ }^{(9,10)}$.

Las principales barreras incluyen acceso limitado a la información sobre la historia clínica del paciente y al sistema sanitario ${ }^{(10)}$. Las brechas en la coordinación tanto en las visitas como en la comunicación entre los profesionales de atención primaria y los oncólogos podrían dar lugar a retrasos en el tratamiento y la atención de seguimiento apropiados ${ }^{(7)}$. Transmitir coordinación entre los equipos profesionales es el único modo de evitar que se genere miedo, incertidumbre y dudas sobre el tratamiento en los pacientes.

\section{c) Accesibilidad a los servicios/atención}

Se considera un factor crítico en la experiencia de los pacientes, centrado en la percepción de la persona respecto al acceso a los servicios sanitarios, incluyendo la atención y cuidados necesarios, la adherencia al seguimiento y la puntualidad en la atención recibida ${ }^{(11,12)}$.

Sería clave para la mejora de la experiencia del paciente la revisión de los sistemas, procesos y operaciones que se necesitan realizar para acceder a los cuidados de su salud o a los tratamientos y reducirlos. Los procesos deben ser amigables y centrados en la percepción de calidad, calidez y empatía que demanda y necesita el paciente y su familia. También son muy importantes en este bloque la señalética, el acceso a transporte público, parking, etc., que son aspectos no sanitarios pero que conforman una percepción global de la atención y cuidado que afecta a la práctica clínica e indudablemente a la experiencia del paciente.

\section{d) Percepción del paciente respecto a la calidad de la atención}

Este factor incluye tanto la percepción de los pacientes en relación a la calidad del tratamiento, como a la calidad de la atención prestada por parte de los profesionales sanitarios, principalmente el médico (identificándose variables como la lealtad, cuidado personal, confianza, continuidad...), incluyendo la calidad del cuidado en el final de la vida. En este sentido se abordan cuestiones como el bienestar y la dignidad, adecuada información y comunicación, apoyo espiritual y emocional, acceso a servicios de apoyo antes y después de la muerte del paciente ${ }^{(4)}$. 


\section{e) Otros aspectos de la experiencia del paciente}

Este último apartado hace referencia a otros temas relevantes como son la accesibilidad del paciente a ensayos clínicos, la prescripción de la medicación y uso de nuevos tratamientos, así como la transformación digital de los sistemas y operaciones, aspecto íntimamente ligado a personalización del servicio al paciente. La comunicación virtual en tiempo real y la intervención en remoto de especialistas podría ser el presente y sin duda el futuro de la relación sanitaria.

La mejora de la experiencia del paciente ya no es una opción, sino una necesidad en los centros sanitarios que buscan cada vez más crear un vínculo con el paciente. La calidad y la seguridad médica, por sí solas, no son decisivas en la elección del centro, ni son una garantía de éxito a nivel de reconocimiento y prestigio. Actualmente existen evidencias de que el enfoque de satisfacción de paciente, asociado solamente a la calidad de servicio, no es suficiente para mejorar los resultados sanitarios; y en la misma línea, seguir estándares de calidad ISO en hospitales no necesariamente mejora la satisfacción de los pacientes ${ }^{(13)}$.

\section{La experiencia del paciente en el ámbito de la salud. utilidad clínica}

Una vez analizados los componentes que permiten acercarnos a la definición de experiencia del paciente, se considera oportuno realizar un análisis sobre las principales conclusiones de la literatura científica, con objetivo de poner de manifiesto la importancia y utilidad de este nuevo concepto, tanto para variables relativas a la salud como para aquellas asociadas a la práctica clínica. Sin duda, el enfoque cada vez más utilizado y centrado en la experiencia del paciente, se acompaña necesariamente de un cambio en la práctica clínica hacia un modelo de atención centrada en el paciente.

En este sentido, la revisión sistemática de numerosos estudios realizada por Doyle et al. ${ }^{(14)}$ sitúa la experiencia del paciente como un pilar indispensable a la hora de establecer la calidad de la atención clínica, junto con la efectividad del tratamiento y la seguridad del paciente. De esta forma, la concepción holística de la calidad, basada en estos tres ejes, se considera superior al hecho de valorar cada uno de estos tres elementos por separado o prescindir de cualquiera de ellos.

Con relación a la efectividad del tratamiento y la seguridad del mismo, cabe destacar que se han utilizado diferentes tipos de medidas para determinar su papel en la calidad de la atención clínica y su relación con el concepto de experiencia del paciente. Entre ellas caben destacar variables objetivas tales como estado de salud (indicadores de "mortalidad", "nivel de glucosa en sangre", "infecciones" y "errores médicos"), uso preventivo de los servicios de salud ("cumplimiento de la medicación", "adherencia al tratamiento"), conductas de salud y uso de recursos ("hospitalización", "readmisión", "uso del servicio de urgencias"); así como variables subjetivas de bienestar ("estado de salud", "capacidad funcional", "calidad de vida", "ansiedad").

De esta forma, la calidad de la comunicación médico-paciente junto al acuerdo del paciente sobre la necesidad de dicho tratamiento ${ }^{(15)}$, se asocian con la efectividad del mismo. Diferentes autores ${ }^{(15-19)}$ han evidenciado un mejor uso de los servicios preventivos, como por ejemplo del screening de cáncer de mama, 
colorrectal y cuello uterino; que, a su vez, facilitan el diagnóstico precoz y mejoran los resultados de los tratamientos. Y, finalmente se asocian sobre la seguridad del paciente, entendida como la capacidad activa del paciente en la toma de decisiones sobre sus preferencias respecto a la oferta de tratamiento disponible. A pesar de ser el factor que menos respaldo científico ha recibido, los datos apuntan al papel que desempeña en el compromiso del paciente con su tratamiento junto con un aumento en la probabilidad de resultados positivos esperados ${ }^{(14)}$.

Algunas de las conclusiones que se extraen del trabajo de Doyle et al. ${ }^{(14)}$ se detallan a continuación. En primer lugar, esta revisión permite afirmar que los tres factores interaccionan entre sí, tal y como señalan los autores "existe evidencia de la relación positiva entre la experiencia del paciente, la seguridad del mismo en el abordaje de la enfermedad y la efectividad del tratamiento aplicado". En segundo lugar, entre las recomendaciones referidas a una práctica clínica de mayor calidad se orienta al personal sanitario a que no considere el concepto de experiencia del paciente como un constructo únicamente subjetivo, sino que tiene su realidad objetiva. Por tanto, resulta clínicamente importante e influyente en el bienestar del paciente durante el proceso de enfermedad, en la adherencia al tratamiento recomendado por el clínico, así como en el uso adecuado de recursos sanitarios.

En tercer lugar y último lugar, queda remarcada la necesidad de formación por parte de los profesionales de la salud en habilidades de comunicación y manejo del paciente, ya que aparece como una variable moduladora de los tres elementos claves de la calidad que el paciente percibe sobre la atención clínica recibida.

\section{Experiencia del paciente en población oncológica}

En España, el cáncer es una de las principales causas de morbilidad, con 215.535 casos estimados para el año 2012, 228.482 casos estimados para el año 2017 y una previsión de 315.413 casos para el año 2035, excluyendo los tumores cutáneos no melanomas ${ }^{(20)}$. Según la SEOM, en los últimos 20 años, el número de tumores ha experimentado un crecimiento constante en España debido no sólo al aumento poblacional, sino también a las técnicas de detección precoz y al aumento de la esperanza de $\mathrm{vida}^{(20)}$. Actualmente, el $5 \%$ de la población española es superviviente de cáncer y se estima que 1.600 .000 personas han superado esta enfermedad, lo que hace necesario aumentar la atención sobre las experiencias de los pacientes con cáncer, tanto durante como después del tratamiento. El objetivo de la mejora de la calidad del sistema sanitario debe sin duda no solo mejorar los resultados terapéuticos sino también reducir los costos y mejorar la mejorar la experiencia del cuidado.

Los resultados obtenidos en los estudios con población oncológica arrojan datos similares a los detallados en el apartado anterior. En este sentido, la experiencia del paciente se asocia con un aumento del uso de recursos necesarios de los servicios de prevención y diagnóstico precoz, favoreciendo la participación de la población en campañas de screening de cáncer colorrectal, cuello uterino y mama ${ }^{(19)}$, así como con mayores tasas de cirugías como tratamiento oncológico de elección ${ }^{(21)}$.

Otras variables relevantes para el ámbito de la salud con las que se asocia la experiencia del paciente con cáncer son el número de visitas al especialista y tiempos de recuperación ${ }^{(22)}$; mejora del estado de la salud general ${ }^{(16)}$; cumplimiento 
con el tratamiento de radioterapia en cáncer de mama y próstata; mejora del estado funcional y de los niveles de ansiedad tras la radioterapia en pacientes con cáncer de próstata $^{(23)}$. También se ha encontrado relación con el número de hospitalizaciones, duración de la estancia durante el ingreso, visitas a urgencias, número de días en unidades de cuidados intensivos, visitas preventivas y medicamentos preventivos de dolor en cáncer de mama y tumores de cabeza y cuello ${ }^{(24)}$.

En la misma línea de hallazgos, el cuidado de la comunicación y la realización de preguntas con respeto y dignidad en pacientes oncológicos, se ha asociado con un mejor control del dolor y una menor tasa de repetición de pruebas innecesarias ${ }^{(25)}$. $\mathrm{Y}$ un dato de elevado interés clínico, es la presencia de malestar ante situaciones en las que se manifiestan aspectos negativos de la experiencia del paciente o en alguno de los otros dos componentes de la calidad de la atención recibida ${ }^{(26,27)}$.

Respecto al tipo de neoplasia, existen numerosos estudios, además de los citados previamente que ponen de manifiesto la relación entre la experiencia del paciente y adherencia al tratamiento en población con cáncer ginecológico, como por ejemplo cáncer de ovario ${ }^{(28)}$; cambios en hábitos de vida saludable y adherencia al tratamiento en mujeres con cáncer de mama ${ }^{(29,30)}$; así como en el uso continuado del tratamiento hormonal sustitutivo durante el tiempo indicado por el especialista en casos de cáncer de mama ${ }^{(31)}$.

Sin embargo, no en todas las investigaciones se indica la presencia relación entre algunos de los componentes de la experiencia del paciente con cáncer y determinadas variables asociadas a la salud. De esta forma Kinnersley et al. ${ }^{(32)}$ no observaron relación entre la accesibilidad a los servicios y determinadas variables tales como resolución de síntomas, preocupaciones y de la capacidad funcional. En la misma línea, Chang et al. ${ }^{(33)}$ indicaron la ausencia de relación entre el área de comunicación de la información por parte del profesional con la percepción de la calidad de la técnica aplicada.

\section{6. ¿Es el enfoque centrado en la experiencia del paciente un nuevo desarrollo para la psicooncología?}

No tanto en España, pero si ya en EE. UU., se están creando en los centros sanitarios equipos dedicados a la mejora de la experiencia del paciente. Por ejemplo, la Clínica Mayo dispone de un departamento de Experiencia del Paciente, y como ella, un elevado porcentaje de hospitales estadounidenses cuentan ya del responsable de la experiencia del paciente cuyo objetivo principal es definir y poner en marcha iniciativas que mejoren la satisfacción de los pacientes y, no menos importante, sino clave, medir y realizar seguimiento de cómo estas iniciativas tienen impacto en los resultados a través de la recopilación de datos y analíticas.

Como consecuencia del aumento creciente de la aplicación de la Psicología de la Salud al cáncer ${ }^{(34,35)}$, la implicación de los psicooncólogos en la estrategia centrada en la mejora de la experiencia del paciente puede llegar a ser un área clave en su actuación. La Psicooncología es un campo interdisciplinar de la psicología y las biomédicas dedicado a la prevención, diagnóstico, evaluación, tratamiento, rehabilitación, cuidados paliativos y etiología del cáncer, así como a la mejora de las competencias comunicativas y de interacción de los sanitarios, además de la optimización de los recursos para promover servicios oncológicos eficaces y de 
calidad. Por tanto, incluye en su definición áreas claves que remarcan el papel activo de esta disciplina y de los profesionales así formados en la mejora de la experiencia del paciente con cáncer ${ }^{(36)}$.

Los objetivos de la Psicooncología van desde la intervención aplicada al paciente, las familias y el personal sanitario, a la prevención e investigación de la influencia de factores psicológicos relevantes en el proceso oncológico, e incluyen la optimización de la mejora del sistema de atención sanitaria. El psicooncólogo es un profesional, en continua evolución y actualmente los nuevos desarrollos centrados en el paciente refuerzan este planteamiento, convirtiendo la línea de actuación centrada en la experiencia del paciente en una nueva oportunidad de desarrollo y actuación profesional.

El profesional responsable de la estrategia centrada en la experiencia del paciente con cáncer en un entorno sanitario debe ser un profesional con capacidad de entender la cultura organizativa del centro, de la relación entre los equipos asistenciales y los profesionales implicados en la atención al paciente. Asimismo, debe ser un profesional formado para gestionar actitudes, comportamientos y maneras de relacionarse tanto del paciente como de los profesionales implicados en su atención. Es imprescindible una visión analítica para saber encontrar información importante de la voz del paciente a través de diversas fuentes como las encuestas, grupos focales, quejas y reclamaciones, monitorización online, cuestionarios de calidad de vida, etc. Y finalmente debe ser un especialista centrado en las dinámicas emocionales, cognitivas y conductuales del paciente y su familia, sin olvidarse del cuidado al profesional.

Este reciente desarrollo permite ofrecer una nueva línea de actuación al profesional de la psicooncología. Indudablemente, queda mucho camino por recorrer para saber incorporar en nuestro país el enfoque de la Experiencia del Paciente en la mejora de un servicio, proceso o ruta asistencial con una adecuada gestión de los recursos. El psicooncólogo es un especialista formado en las principales metodologías y técnicas de implicación y empoderamiento de pacientes, es capaz de obtener patient insights, trabajar las relaciones y los entornos saludables para mejorar la experiencia del paciente y finalmente está entrenado para concebir, planificar y desarrollar un proyecto que tiene como objetivo la mejora de la experiencia del paciente.

\section{Conclusiones}

La Experiencia del Paciente no es una moda, sino un cambio real en la manera de trabajar en las organizaciones sanitarias, como lo fue la calidad o como lo es la telemedicina.

La práctica médica siempre ha definido la calidad y la excelencia en términos de resultados clínicos (ratios de curación, remisión, complicaciones, mortalidad...), que son tan sólo la mitad de la ecuación, pues la salud está sujeta a una constelación de sentimientos y percepciones que no podemos soslayar. Aquí es donde entra en escena la experiencia de paciente. Un paciente puede carecer de los conocimientos necesarios para evaluar la conveniencia de un procedimiento médico, sin embargo, por muy perdido que se encuentre, siempre podrá juzgar con claridad lo buena o mala que ha sido su experiencia. 
Es un cambio cultural y como todos los cambios importantes en sanidad, el protagonista y el centro es el paciente y en torno a él deben girar los profesionales y las instituciones que van a liderar el cambio. La estrategia centrada en la experiencia del paciente debe estar en el ADN de cada uno de los profesionales que trabajan en la institución. Las principales áreas de actuación del responsable de experiencia del paciente irán desde gestionar la cultura del centro sanitario, definir estrategias de experiencia y su mejora hasta la gestión de quejas, incluyendo gestión de calidad y mejora continua. Asimismo, debe incluir la atención a las familias y entorno más cercano de los pacientes. Las mediciones y métricas son otra prioridad, seguida de la mediación o "advocacy", es decir un papel defensor del paciente ante la organización sanitaria. El psicooncólogo puede desempeñar este papel clave absolutamente involucrado en la estrategia, liderazgo y gobierno de la organización sanitaria.

\section{Referencias bibliográficas}

1. Wolf JA, Niederhauser V, Marshburn D, and LaVela SL. Defining Patient Experience. Patient Exp J 2014; 1: 7-19.

2. The Beryl. Institute [Internet]. Defining Patient Experience. [consultado 17 de febrero de 2018]. Disponible en: http://www.theberylinstitute.org/?page=DefiningPatientExp

3. Mollica MA, Lines LM, Halpern MT, Ramirez E, Schussler N, Urato M, Wilder S, and Kent EE. Patient experiences of cancer care: scoping review, future directions, and introduction of a new data resource: Surveillance Epidemiology and End ResultsConsumer Assessment of Healthcare Providers and Systems (SEER-CAHPS). Patient Exp J 2017; 4: 103-21.

4. Mazor KM, Beard RL, Alexander GL, Arora NK, Firneno C, Gaglio B et al. Patients' and family members' views on patient-centered communication during cancer care. Psychooncology 2013; 22:2487-95. Doi: 10.1002/pon.3317

5. Baile WF, Aaron J. Patient-physician communication in oncology: past, present, and future. Curr Opin Oncol 2005. 17:331-5. Doi: 10.1097/01.cco.0000167738.49325.2c

6. Lewis R, Neal RD, Williams NH, France B, Wilkinson C, Hendry M et al. Nurse-led vs. conventional physician-led follow-up for patients with cancer: systematic review. J Adv Nurs 2009; 65:706-23. doi: 10.1111/j.1365-2648.2008.04927.x

7. Shen MJ, Binz-Scharf M, D’Agostino T, Blakeney N, Weiss E, Michaels M et al. A mixedmethods examination of communication between oncologists and primary care providers among primary care physicians in underserved communities. Cancer 2015; 121: 908-15. doi: $10.1002 /$ cncr.29131.

8. Young JM, Butow PN, Walsh J, Durcinoska I, Dobbins TA, Rodwell L, et al., Multicenter randomized trial of centralized nurse-led telephone-based care coordination to improve outcomes after surgical resection for colorectal cancer: the CONNECT intervention. $\mathrm{J}$ Clin Oncol 2013; 31: 3585-91. doi: 10.1200/JCO.2012.48.1036.

9. Walsh J, Young JM, Harrison JD, Butow PN, Solomon MJ, Masya L, et al. What is important in cancer care coordination? A qualitative investigation. Eur J Cancer Care (Engl) 2011; 20: 220-7. doi: 10.1111/j.1365-2354.2010.01187.x.

10. Hawley ST, Janz NK, Lillie SE, Friese CR, Griggs JJ, Graff JJ et al. Perceptions of care coordination in a population-based sample of diverse breast cancer patients. Patient Educ Couns 2010; 81 Suppl: 34-40. 
11. Carpentier MY, Vernon SW, Bartholomew LK, Murphy CC, Bluethmann SM. Receipt of recommended surveillance among colorectal cancer survivors: A systematic review. J Cancer Surviv 2013; 7: 464-83. doi: 10.1007/s11764-013-0290-X.

12. Kimman ML, Bloebaum MM, Dirksen CD, Houben RM, Lambin P, Boersma LJ. Patient satisfaction with nurse-led telephone follow-up after curative treatment for breast cancer. BMC Cancer 2010; 10: 174. doi: 10.1186/1471-2407-10-174.

13. Keshtkar V, Bazgir M, Anvar M. Does implementation of ISO standards in hospitals improve patient satisfaction? J Health Man \& Info 2017;4:7-11.

14. Doyle C, Lennox L, Bell D. A systematic review of evidence on the links between patient experience and clinical safety and effectiveness. BMJ Open 2013; 3:e001570. doi:10.1136/bmjopen-2012- 001570.

15. O'Malley AS, Sheppard VB, Schwartz M, et al. The role of trust in use of preventive services among low-income African-American women. Prev Med 2004; 38: 777-85.

16. Kaplan SH, Greenfield S, Ware JE. Assessing the effects of physician-patient interactions on the outcomes of chronic disease. Med Care 1989; 27(3 Suppl): 110-27.

17. Flocke SA, Stange KC, Zyzanski SJ. The association of attributes of primary care with the delivery of clinical preventive services. Med Care 1998; 36: AS: 21-30.

18. Carcaise-EdinboroP,Bradley CJ.Influence of patient-providercommunication on colorectal cancer screening. Med Car 2008; 46: 738-45. doi: 10.1097/MLR.0b013e318178935a.

19. Sequist TD, Schneider EC, Anastario M, Odigie EG, Marshall R, Rogers WH et al. Quality monitoring of physicians: linking patients' experiences of care to clinical quality and outcomes. J Gen Intern Med 2008; 23: 1784-90. doi: 10.1007/s11606-008-0760-4.

20. Sociedad Española de Oncología Médica. [Internet]. Las cifras del cáncer en España 2018. SEOM. [Acceso 18 de febrero de 2018]. Disponible en: https://seom.org/seomcms/ images/stories/recursos/Las_Cifras_del_cancer_en_Espana2018.pdf

21. Saultz JW, Lochner J. Interpersonal continuity of care and care outcomes: a critical review. Ann Fam Med 2005; 3: 159-66.

22. Blasi ZD, Harkness E, Ernst E, et al. Influence of context effects on health outcomes: a systematic review. Lancet 2001; 357: 757-62.

23. Stewart MA. Effective physician-patient communication and health outcomes: a review. Can Med Assoc J 1995; 152: 1423-33.

24. Cabana M, Jee S. Does continuity of care improve patient outcomes? J Fam Pract 2004; 53: 974-80.

25. Agoritsas T, Bovier PA, Perneger TV. Patient reports of undesirable events during hospitalization. J Gen Intern Med 2005; 20: 922-8. doi: 10.1111/j.1525-1497.2005.0225.x

26. Weingart SN, Pagovich O, Sands DZ, Li JM, Aronson MD, Davis RB, et al. What can hospitalized patients tell us about adverse events? Learning from patient-reported incidents. J Gen Intern Med 2005; 20: 830-6. doi: 10.1111/j.1525-1497.2005.0180.x

27. Weissman JS, Schneider EC, Weingart SN, Epstein AM, David-Kasdan J, Feibelmann S, et al. Comparing patient-reported hospital adverse events with medical record review: do patients know something that hospitals do not? Ann Intern Med 2008; 149: 100-8. doi: 10.7326/0003-4819-149-2-200807150-00006

28. Stevenson FA, Cox K, Britten N, et al. A systematic review of the research on communication between patients and health care professionals about medicines: the consequences for concordance. Health Expect 2004; 7: 235-45. doi: 10.1111/j.13697625.2004.00281.x 
29. Arbuthnott A, Sharpe D. The effect of physician-patient collaboration on patient adherence in non-psychiatric medicine. Patient Educ Couns 2009; 77: 60-7. doi: 10.1016/j. pec.2009.03.022.

30. Safran DG, Miller W, Beckman H. Organizational dimensions of relationhip-centred care. J Gen Intern Med 2005; 21(Sppl1): S9-15.

31. Kahn KL, Schneider EC, Malin JL, Adams JL, Epstein AM. Patient centered experiences in breast cancer: predicting long-term adherence to tamoxifen use. Med Care 2007; 45: 431-9. doi: 10.1097/01.mlr.0000257193.10760.7f

32. Kinnersley P, Stott N, Peters TJ, Fitzpatrick B, Little P, Watt GC, Mercer SW. The patientcentredness of consultations and outcome in primary care. Br J Gen Pract 1999; 49: 7116. doi: 10.3399/bjgp12X653633.

33. Chang JT, Hays RD, Shekelle PG, MacLean CH, Solomon DH, Reuben DB, Roth CP, et al. Patients' global ratings of their health care are not associated with the technical quality of their care. Ann Intern Med 2006; 144: 665-72. doi: 10.7326/0003-4819-144-9200605020-00010

34. Bayés R. Psicología oncológica. Barcelona: Martínez Roca; 1990.

35. Holland JC. Psycho-Oncology. Nueva York: Oxford; 1998.

36. Cruzado JA. La formación en psico-oncología. Psicooncología 2003; 0: 9-19. 